\title{
CONTENIDOS ENRIQUECIDOS PARA NIÑOS O LAS NUEVAS FORMAS DE LEER, CREAR Y ESCUCHAR HISTORIAS: UNA PROPUESTA DE CLASIFICACIÓN
}

\author{
Araceli García-Rodríguez \\ Universidad de Salamanca \\ araceli@usal.es \\ Raquel Gómez-Díaz \\ Universidad de Salamanca \\ rgomez@usal.es
}

\section{RESUMEN / ABSTRACT}

Una de las características del mundo digital infantil es la riqueza y diversidad de contenidos que pueden ir, de lo meramente textual y gráfico, a ofrecer productos enriquecidos con vídeo, sonido, movimiento... con los que se puede interactuar y que sirven para leer, escuchar historias, crearlas o jugar con ellas. Ante tal variedad es necesario conocer cuáles son sus características principales, así como realizar una sistematización que facilite su utilización por parte de los mediadores. Este artículo propone una clasificación de los contenidos digitales para niños y sus enriquecimientos atendiendo a diferentes factores e incluyendo en cada uno de ellos títulos recomendados.

PALABRAS ClAVE: literatura digital infantil; lectura digital infantil; aplicaciones de lectura para niños; libros enriquecidos; clasificación de aplicaciones de lectura infantil.

One of the characteristics of the digital world for children is the richness and diversity of content that can range from the merely textual and graphic, to the availability of products enriched by video, sound, movement ... with which you can interact and that are useful for reading stories, as well as listening, creating, or playing with them. Given this variety, it is necessary to know what their main features are and how to systematize them in order to facilitate their use for mediators. This article proposes a classification of digital content for children and its improvement according to different factors and including for each recommended titles.

KEYWORDS: Children's Digital Literature; Children's Digital Reading; Children's reading app; Enhanced books; children's reading app taxonomy. 


\section{INTRODUCCIÓN}

Como afirma Cassany (269), la lectura no es algo natural, sino una práctica social que cambia en cada momento de la historia, en cada comunidad y en cada contexto, aunque los textos sean los mismos. En el momento actual la lectura está fuertemente influida por la tecnología móvil que está provocando cambios en la manera de leer y de desplazarse por el texto, está ofreciendo nuevas posibilidades en función de las necesidades, gustos y prioridades de los lectores. Surge así la ciberliteratura (Morales 96), como una nueva propuesta estética que requiere de la pantalla para poder acceder a ella, y ante las nuevas posibilidades los hábitos lectores cambian, fijándose patrones de comportamiento que afectan a los modos de consumo de la información en general y de la lectura en particular.

Los primeros libros electrónicos no estaban adaptados al público infantil debido a que el uso de la tinta electrónica no permite los colores, ni la interacción, pero estas limitaciones se han visto rápidamente superadas con las tabletas que permiten que los más pequeños se vayan acercando poco a poco a la lectura digital, aunque ello esté provocando discusiones sobre la idoneidad de que los niños utilicen estos dispositivos, bien en su tiempo de ocio o en la escuela. Y es que la lectura digital suscita todo tipo de preguntas entre los padres y adultos mediadores (profesores, padres y bibliotecarios) involucrados en la educación lectora, de ahí que en los últimos años se hayan publicado diferentes guías sobre las buenas prácticas en la literatura digital, como la elaborada por el grupo francés Bayard Jeunesse, la realizada por el Joan Ganz Cooney Center en 2015 o la web Literacy Apps <http:// literacyapps.literacytrust.org.uk/> creada por el El National Literacy donde se recomiendan aplicaciones que contribuyen al desarrollo de las habilidades de lenguaje y comunicación en niños menores de cinco años.

Sin menospreciar la necesidad de este tipo de discusiones, no es el objetivo de este trabajo tratar si es adecuado o no el uso de dispositivos, o las ventajas e inconvenientes de la lectura digital en los niños, sino ofrecer una propuesta de clasificación de un tipo de productos, que, tal como afirman Meyers, Zamimpaima y Frederico (916), han marcado un hito importante en la atracción de los niños hacia las historias.

Las expectativas que genera lo digital quedan patentes en la cantidad de libros editados en el extranjero que ofrecen versión en español (Ruiz-Domíngez 234), en el aumento de la producción en español, en la creación de empresas especializadas en el desarrollo de contenidos digitales y en la aparición de 
premios y sellos de calidad como KAPi Award for Best Children's Ebook $<$ http://kapiawards.com/>, FutureBook Innovation Awards <http://www. thebookseller.com/> o el premio a la mejor aplicación en la Feria del libro de Bolonia (Italia) <http://www.bookfair.bolognafiere.it/>.

El mercado de los contenidos digitales para niños está en expansión y las aplicaciones para dispositivos táctiles cubren espacios de ocio diferentes, entre ellos la lectura, que pueden categorizarse de muy diversas formas, por lo que es cada vez más necesario establecer una sistematización de los contenidos.

\section{JUSTIFICACIÓN Y OBJETIVOS}

El desarrollo vertiginoso del mundo digital hace más necesaria que nunca la aparición de estudios que ayuden a conocer y entender esta nueva realidad. Se necesitan descripciones y clasificaciones de un corpus que también reclama análisis en relación con sus características específicas; los hábitos y las habilidades lectoras que potencia; las modificaciones que puede estar implicando la lectura (Alonso-Arevalo y Cordón-García, 25-47); los criterios y parámetros de selección (García-Rodríguez y Gómez Díaz, Las demasiadas aplicaciones 2-17) o sus posibilidades didácticas. Solo de esta forma podremos reducir la brecha existente entre la atracción que las aplicaciones ejercen sobre los más pequeños y el desconocimiento de las mismas por parte de los mediadores (Turrión Penelas 15).

Por tanto, necesitamos crear y definir términos y categorías específicas y diferenciadas para la literatura infantil y juvenil digital (en adelante LIJD), que se adapten a la realidad de lo que el mercado nos ofrece, dando a conocer sus posibilidades.

Desde que la tecnología irrumpió en el mundo del libro, la variedad y diversidad de productos provocan confusión en qué es y qué no es un libro electrónico al no estar claramente delimitada una tipología de contenidos digitales, como sí ocurre en el caso del libro impreso. Por ello, los objetivos de este artículo son: establecer una clasificación de contenidos digitales para niños, atendiendo a diferentes factores, que permitirá un mayor conocimiento de este entorno cambiante y definir los diferentes tipos de enriquecimientos de las obras digitales infantiles, aportando, en todos los casos, recomendaciones de productos especialmente interesantes. 


\section{METODOLOGÍA}

Además de la exploración de la literatura relativa al tema extraída de las bases de datos tanto generales (Scopus y Wos) como especializadas (ERIC, LISA, LISTA, DIALNET), se han consultado distintos blogs como el de Elisa Yuste $<$ http://www.elisayuste.com/>, Canal Lector $<$ http://www.canallector.com/>, Literaturas Exploratorias $<\mathrm{https}$ ://literaturasexploratorias.com/ $>$, Anatomía de la Edición <http://anatomiadelaedicion.com/>, Dosdoce < http://www. dosdoce.com/>, etc. y webs especializadas en aplicaciones infantiles como BestappsforKids $<$ http://www.bestappsforkids.com/ $>$, Bibapps $<$ http://bibapps. com/>, Boolino <http://www.boolino.es/>, Frikids <http://www.frikids. com/>, Smartappsfokids <http://www.smartappsforkids.com/>, La Souris Grise $<$ http://www.souris-grise.fr/>, Generación App $<$ http://generacionapps. $\mathrm{com} />$ o Proyecto Guappis $<$ http://proyectoguappis.blogspot.com.es/>, entre otros, que han permitido conocer el estado de la cuestión sobre el tema y seleccionar un conjunto de aplicaciones y de contenidos. Posteriormente, se realizó su descarga y se procedió a la sistematización de la información para poder seleccionar los más adecuados en cada una de las categorías ${ }^{1}$.

\section{HACIA UNA TIPOLOGÍA DE LA LIJD}

Una de las características del mundo digital infantil es la riqueza y diversidad de contenidos que pueden ir de lo meramente textual y gráfico, a ofrecer productos enriquecidos con vídeo, sonido, movimiento... con los que se puede interactuar, pero también su gran variabilidad, lo que dificulta en gran manera una sistematización estable y única.

Tal como afirma Ruiz-Domíngez (2014, p. 234), cuando nos aproximamos a la variedad de aplicaciones de cuentos infantiles que tenemos a nuestra disposición, descubrimos la complejidad que supone establecer un criterio para su clasificación. Entre otras posibilidades, podríamos atender a criterios que tienen que ver con la tipología de los textos literarios digitales (Borrás, 2005), con su procedencia (Koskima, 2005), con el grado de participación

Para completar este listado se recomienda la consulta de García-Rodríguez y GómezDíaz, Niños y apps en el que se recoge una amplia selección y categorización de aplicaciones para lectoescritura. 
e interacción con el lector (Moreno,2002; Ryan, 2004) o bien centrarnos en las prestaciones interactivas y multimedias que se han puesto a disposición del texto e incluso atendiendo a criterios de $\operatorname{edad}^{2}$ o tema como se hace en la literatura impresa, pero en el caso de los contenidos digitales, necesitamos una clasificación específica.

Por ello, con el objetivo de trazar las líneas que permitan definir una clasificación terminológica, se establecen, en primer lugar, tres grandes categorías, libro electrónico (contenidos), aplicaciones de lectura y libro app. Cada categoría está formada por varias subcategorías en función de diferentes aspectos: contenido, requisitos de acceso y objetivos.

2 La edad, junto a otros aspectos como el género, es un factor que influye en la forma en la que se lee un libro electrónico, según un estudio sobre el comportamiento de los lectores llevado a cabo por Jellybooks. http://www.elisayuste.com/edad-factor-para-finalizar-un-libro/ 


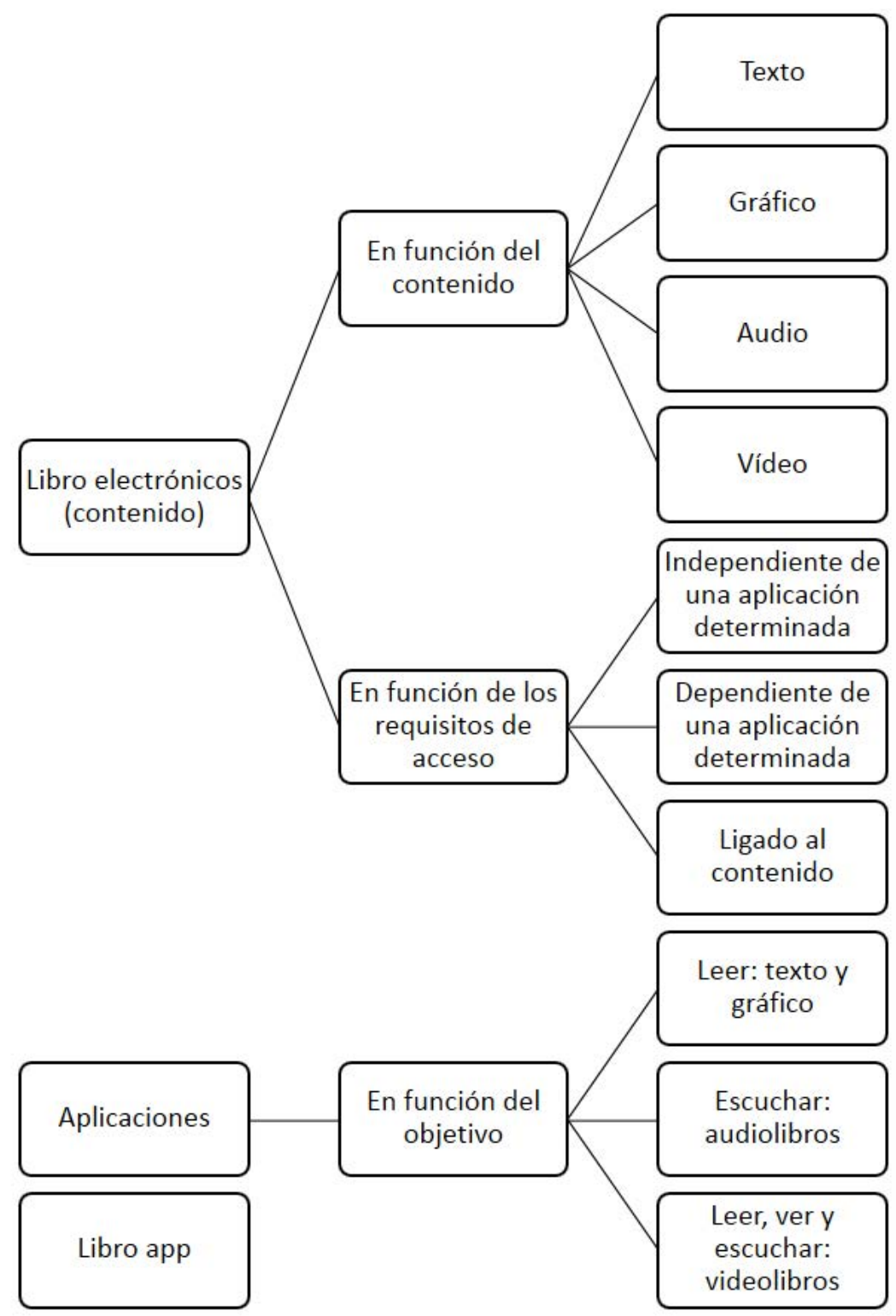

Fig. 1. Tipología de la literatura infantil digital Elaboración propia 


\section{Libro electrónico (contenido)}

Dentro de esta categoría, Koskimaa (85-88) distingue entre los textos que originalmente se publicaron de forma impresa y que más tarde se digitalizaron (textos digitalizados), los que se publican al mismo tiempo en papel y electrónico, los que han surgido en formato digital para producir efectos que no son posibles en los textos impresos (textos digitales programados) $\mathrm{y}$, por último, textos que hacen uso de los recursos de internet (webtextos).

Lucía Mejías (116) y Escandell (74) establecen tres tipos de textos digitales: aquellos que tienen un origen manuscrito o impreso y se convierten a lo digital mediante un proceso de escaneado; los que se han creado en un entorno electrónico (por ejemplo, con procesadores) pero siguen los patrones de conceptualización de páginas, y los que son puramente digitales, como, por ejemplo, un objeto animado e integrado y cuya trasposición al papel no sería posible sin la pérdida de información. Por otro lado, Yokota (578) distingue entre el escaneo de libros y la transformación en algo similar al vídeo; aquellos que incluyen características propias de lo digital (por ejemplo la función del giroscopio) y los que incluyen interactividad.

En nuestro caso se hace una doble clasificación, en función del contenido y del sistema de acceso. En el primer caso los libros electrónicos pueden ser:

- Texto. Se incluyen en esta categoría muchos contenidos que tuvieron un origen analógico, es decir, los libros digitalizados, en los que la única transformación respecto del papel era el cambio de soporte. En ellos suele haber casi exclusivamente texto, o como mucho, imágenes fijas no redimensionables, por lo que sus posibilidades de interactividad son bastante limitadas, siempre dependiendo de la aplicación utilizada. Un ejemplo de este tipo de obras pueden ser los cuentos recogidos en la Biblioteca Digital Internacional para niños $<$ http://es.childrenslibrary. $\operatorname{org} />$.

En los primeros libros electrónicos, la tecnología lo único que hacía era permitir la personalización del texto y por esta se entendía el cambio del tamaño y tipo de letra. Poco a poco se fue incluyendo un mayor aprovechamiento de las potencialidades de la tecnología permitiendo la inserción de vídeo como en álbum de Sebastián Pérez y Benjamin Lacombe, El herbario de las hadas, audio objetos animados... que invitan a que el lector configure e interactúe con la historia convirtiendo su experiencia de lectura en algo único e irrepetible. 
- Gráfico (cómic y revistas). Tanto en los cómics como en las revistas lo gráfico tiene gran importancia, incluso más que el texto pues en el primer caso las historias están explicadas en viñetas, mientras que en el segundo el texto y la imagen se van alternado.

- Audiolibros. Son locuciones narradas o leídas de libros ya existentes o creados expresamente para ser difundidos en este formato audible y que, en ocasiones, pueden acompañarse con música y efectos de sonido. Se pueden escuchar en ordenadores, tabletas, teléfonos inteligentes, iPod, reproductores de MP3, etc. ${ }^{3}$ Hay que distinguir entre aquellos que han sido creados directamente con una configuración específica para ser escuchados, y los derivados, que son aquellos que no se hicieron inicialmente como audiolibros, pero que mediante plugin se pueden convertir en sonido en algunas aplicaciones. (García-Rodríguez, Gómez-Díaz, Niños y lectura digital 34). Un ejemplo de audiolibros especialmente recomendables son los editados por Penguin, muchos de ellos leídos y dramatizados por actores de reconocido prestigio. $<$ https:// www.penguin.co.uk/puffin/search/?_charset_=UTF-8\&q=audiobooks $>$.

- Videolibros. Son grabaciones que reproducen con imagen y audio el contenido de un libro. Dentro de este término podríamos incluir:

- Libros que permiten la reproducción en forma de vídeo, sin pausa, de diferentes historias, algo habitual en los títulos dirigidos a los más pequeños.

- Libros disponibles a través de plataformas de vídeo como Youtube o Vimeo, normalmente reproducciones animadas que se pueden descargar directamente desde la red. Existen incluso algunas empresas que disponen de canales específicos como KidsKioske ${ }^{4}$.

Por lo que se refiere a la clasificación en función de los requisitos de acceso nos encontramos:

- Los que son independientes de una aplicación. Estos contenidos se pueden comprar en una librería digital o descargar de una biblioteca

3 Se recomienda la consulta de la Asociación de Editores de audiolibros (APA) https:// www.audiopub.org y Me gusta escuchar de Penguin Randon House http://megustaescuchar. com/

http://www.youtube.com/user/Kidskioske 
digital, como el Proyecto Gutenberg después leer con una aplicación general como Ibooks; BlueFire, Kindle...

- Los dependientes de una aplicación determinada. En este caso, la aplicación se conecta a una tienda cuyos contenidos solo pueden ser leídos a través de esa aplicación como ocurre con PlayTales.

- Los ligados al contenido (libros-app). En esta categoría se incluyen aquellos libros que aunque puedan estar ligados a la tienda de una aplicación determinada, se pueden comprar también de manera independiente como "Por Cuatro esquinitas de nada" que se puede adquirir dentro de la aplicación de lectura Play Tales o de forma individual en App Store (García-Rodríguez; Gómez-Díaz Niños y lectura digital: 30 ).

\section{Aplicaciones de lectura}

Una app es una aplicación de software que se instala en un dispositivo móvil y que permite a los usuarios realizar una labor concreta, en el caso que nos ocupa, leer, de modo que una app de lectura permitirá el acceso a contenidos determinados con el objeto de leerlos, crear una historia, escucharlos...

Al igual que en caso de los libros electrónicos se puede establecer una clasificación en función del tipo de acceso u otros factores, pero también de la acción que queramos hacer con ella, en el caso de los niños: leer, escuchar, crear y jugar.

\section{Leer}

Si entendemos el concepto de lectura como algo amplio y no la simple decodificación del texto escrito, en este grupo de aplicaciones se incluyen aquellas que facilitan el acceso a los libros, ya sean textuales, con un mayor contenido gráfico, como son los cómics, o incluso audios. Se pueden mencionar en este caso The tales of Beatrix Potter, Play Tales, Mis cuentitos, Joy Tales, Genius Books, Sesame Street, eBook, Kidint, StoriesAlive o StoryTime.

\section{Crear}

Desde siempre los niños han tenido una atracción especial por escuchar y crear historias. Gracias a las tabletas y los smartphones esas historias trascienden de lo textual $\mathrm{u}$ oral y encontramos aplicaciones que permiten la creación de obras originales y personalizadas, en las que además se pueden grabar vídeos, incorporar música, utilizar nuestra propia voz para construir 
un cuento e incluso introducir realidad aumentada a través de aplicaciones como Aurasma. Precisamente esto es lo que permiten hacer las aplicaciones denominadas storytelling, en las que el lector se convierte en autor ${ }^{5}$, dentro de las cuales se pueden distinguir:

- Aquellas que permiten la escritura de cuentos a través de ilustraciones e incluso textos ya precargados en la aplicación o incorporados por el propio lector. Destacamos Story Time, Play Kids, Cuentacuentos para niños, Imagistory y In my dream.

- Las que trabajan con animaciones, vídeos, música y la creación de libros, películas animadas y videocuentos. En esta categoría se pueden mencionar Easy Stop Motion, Toontastic, Toontastic Jr. Pirates y Animation Studio.

- Las que sirven para crear cómic (cómic marker), como Comic Star, Comic Marker, Make Beliefs Comix o Strip Designer.

\section{Escuchar}

$\mathrm{Al}$ igual que ocurre con el resto de los contenidos digitales, existen aplicaciones específicas para audiolibros, que generalmente se adquieren bajo el modelo de descarga gratuita con compras integradas, como Dahl Audio y Audiobook Kids.

\section{Ver y escuchar}

En esta categoría están las aplicaciones específicas para los videolibros, es decir, grabaciones que reproducen contenidos con imagen y audio, que pueden ser de gran utilidad para los que todavía no saben leer o para el aprendizaje de idiomas, porque en este caso, a diferencia de los audiolibros tienen el refuerzo de la imagen. Es el caso de Vivaz Books o Stories Alive.

\section{Libro app}

Un libro app es aquel en el que el contenido no se puede desligar de la aplicación y, a diferencia de las apps de lectura, son títulos concretos para los que se ha desarrollado un programa específico (Gómez-Díaz et al. 73)

No se consideran storytelling aquellas aplicaciones que permiten simplemente grabar con nuestra voz una historia por ser una funcionalidad habitual en las aplicaciones de lectura dirigidas a los más pequeños. 
Al igual que los libros impresos se pueden clasificar en función de su contenido: ficción, informativo; del diseño, ilustrado, de imágenes, etc., pero en el caso de los digitales también debemos conocer el tipo de enriquecimiento. Por ello dedicamos el siguiente apartado a conocer los diferentes tipos de enriquecimientos, siempre teniendo en cuenta que la mezcla de estos recursos es habitual en los libros infantiles y que por lo tanto un mismo libro puede combinarlos.

\section{EL ENRIQUECIMIENTO EN LOS CONTENIDOS INFANTILES}

Un contenido es enriquecido cuando además de tener información textual, esta se combina con información multimedia; es decir, audio, imagen en movimiento, enlaces hipertextuales, mapas, juegos e incluso herramientas de participación en las redes sociales, ya sea a otras partes del documento o fuera de él, algo que se conoce habitualmente como interactividad.

La interactividad permite al lector tener una experiencia única que rompe la linealidad y la secuencialidad de la lectura analógica, ya que es él quien puede decidir el orden en el que se mueven los objetos, si suena o no la música, si lo lee o se lo leen... (Cahill 36). La posibilidad de realizar diferentes acciones es lo que acrecienta más aún la experiencia única de la lectura digital, pero el enriquecimiento siempre debe estar al servicio de la historia, ya que, aunque estos nuevos componentes atraen a los niños, su uso inapropiado puede distraer de la narración (Meyers, E. M., Zaminpaima, E., \& Frederico, A.: 916) y por tanto entorpecer la comprensión de la historia (Cahill: 36).

Los contenidos pueden tener distinto grado de interactividad, desde una interacción muy sencilla a la integración de elementos con realidad aumentada o la personalización de los personajes. Lo importante es que éste tenga sentido y no sea un mero adorno de la historia.

Los enriquecimientos más habituales se analizan en los siguientes apartados.

\section{Enlaces hipertextuales}

Consiste simplemente en el establecimiento de enlaces que permiten navegar por las distintas partes de la obra, fichas de personajes, mapas, ilustraciones, vídeos, etc.. o enlaces externos.

Un ejemplo es El reino subterráneo, basado en los que consideraron los primeros libros interactivos, Elige tu propia aventura de Timun Mas, donde 
el lector puede construir diferentes historias en función de la página que seleccione al finalizar cada capítulo. Otro ejemplo es Mi vecino de abajo de Daniel Nesquens, que en su versión digital contiene numerosos hipervínculos que permiten que los niños accedan y exploren otros contenidos adicionales, e incluso ¿Quién habrá robado la luna? en el que el hiperenlace es a un conjunto de canciones relacionadas con la historia.

\section{Sonido}

Es muy frecuente que las obras para niños incluyan sonido y dentro de él debemos distinguir entre el que se produce al realizar distintas acciones como tocar los personajes u objetos incorporados en cada página; la banda sonora que puede ser común a toda la obra como ocurre en Lo que comen los ratones, o cambiar en cada página como en Los fantásticos libros voladores de Mr. Morris Lessmore; finalmente la locución de la obra, que incluso puede ser grabada por el propio lector como en Las hadas de la casa Lis o escucharla en varios idiomas o variantes idiomáticas (español de América o de España; inglés británico o americano), en el caso de Play Tales.

Es habitual que estas tres opciones se puedan activar o desactivar bien de forma conjunta o independiente, es decir, se pueda combinar la música, el sonido y la locución, en función de los gustos del lector, que es lo que sucede en Descubriendo las telecomunicaciones con Mobi y Fono.

Otra funcionalidad es la que se incluye en algunas aplicaciones en las que dependiendo de donde se toque se permite activar distintas escalas musicales en los objetos como en la ya mencionada Los Fantásticos libros voladores de Mr Morris.

\section{Movimiento de objetos y personajes}

El movimiento de objetos y personajes activado por el usuario a través del tacto (hotspots) es habitual en los contenidos infantiles. Ejemplos de este tipo de acción se dan en Nurot, Buenas noches Mo o El cabeza de Chanchoo: leyenda de Valparaiso.

\section{Imágenes interactivas}

Son aquellas que se pueden activar por partes, por ejemplo, poner el nombre de un país o ciudad en un mapa político, o las partes de un animal en un libro de zoología, un tipo de enriquecimiento más habitual en los libros informativos y en las aplicaciones educativas. Es el caso de Medusa! HD 
en el que pinchando en el mapa explica las variedades de medusas que hay y de World of Dinnosaurs: Kids en el que algunas imágenes explican las partes de los yacimientos.

\section{Vídeos}

Los vídeos pueden servir de apoyo al texto o estar integrados como una parte más de la historia, pero también muchas obras se pueden convertir en videolibros activando el modo de lectura automática como en los títulos de las aplicaciones de lectura Mis cuentitos o en el libro app A la cama.

\section{Checklist de contenidos}

Algunos libros pueden contener preguntas que sirvan para evaluar el nivel de asimilación y comprensión de la información, normalmente desde un punto de vista lúdico. Un caso interesante son los contenidos de la aplicación Booksy o los de Kidint que incluye preguntas con diferentes niveles de dificultad.

\section{Elementos pop-up}

Se denominan рор-ир los elementos emergentes que se abren al realizar una determinada acción. Un tipo de ellos son las notas emergentes o "pop-up footnotes", que se activan para obtener más información de un determinado objeto, por ejemplo, en la ya citada World of Dinosaurs: kids, en la que al tocar cada dinosaurio aparece información, en Lincoln en la que aparece información sobre el personaje al hacer tap sobre él o en los títulos de StoryTime donde aparece pop-up footnotes de lo que dicen los diferentes personajes.

También hay libros infantiles digitales que se consideran de esta manera cuando ofrecen un efecto tridimensional, como es el caso de Buenas noches Mo, Roxie's Doors o los de Chaguipatrulla basados en la película de animación de Disney Car.

\section{Efectos de focalización}

Dentro de estos efectos se encuentra el efecto lupa, en el que al tocar un objeto se agranda la parte seleccionada como si de una lupa se tratara, como ocurre en World of Dinosaurs: kids, pero también aquel que enfoca e ilumina una sola parte de la pantalla, bien de forma automática o al hacer tap como en ¿Quién habrá robado la luna? o el libro Secretos de la luna, de la aplicación Play Tales. 


\section{Activación por movimiento o por sonido}

Quizá los efectos más novedosos son los que se consiguen al mover el dispositivo como ocurre con el libro de $W u w u \& \mathrm{Co}^{6}$, en el que cuando se mueve el dispositivo se accede al contenido textual o a la imagen, o en el conocido libro de Alice for the iPad.

A través del sonido también se pueden activar determinados componentes; por ejemplo, en El Hada de los sueños. Mr. Sandan, cuando al hacer "sssshh" los personajes se callan y pasa la página, y en $W u w u \& C o$, donde los personajes repiten lo que el lector dice a través del micrófono.

\section{Realidad aumentada}

La realidad aumentada es el conjunto de tecnologías que permite la superposición, en tiempo real, de imágenes, marcadores o información generados virtualmente sobre elementos localizados en el mundo real (Sainz 7), es decir, que la información sobre el mundo real se convierte en interactiva y digital. http://www.realidadaumentada-fundaciontelefonica.com/realidadaumentada.pdf

Existen tres tipos de libros con realidad aumentada: libro en papel con marcadores más aplicación; libro en papel con marcadores que se completa con un programa de ordenador; libro con marcadores incorporados cuyos contenidos en realidad aumentada en 3D se activan a través del gadget de una consola (Gómez-Díaz et al. 99).

Algunas empresas han apostado por dedicarse en exclusiva al desarrollo de este tipo de productos, como es el caso de Baibuk, que ha comenzado a comercializar libros infantiles bajo la marca BooksARalive ${ }^{7}$ con varias colecciones, como Conoce a los animales y otros títulos como El árbol de Navidad, Jack-o-Lantern, Hansel y Gretel. Finalmente se puede mencionar Los fantásticos libros voladores de Mrs. Morris Lessmore en cuyo caso es necesario descargar en App Store la aplicación Imagnotron y Un lobo boquiabierto.

\section{Personalización de la historia}

La literatura electrónica ofrece la posibilidad al lector de "actuar" en la obra y ejercer distintos roles para participar en la historia, de modo que el lector digital 
toma decisiones en el proceso de lectura y por este motivo está comprometido con la obra, y se convierte en autor y lector a la vez (Manresa Potrony 14).

Se pueden distinguir dos tipos de personalización, aquella que Ryan denomina ontológica, porque los usuarios son los que determinan el argumento como ocurre en "El reino subterráneo", y la exploratoria en la que los usuarios son libres de moverse por el libro pero sus movimientos no hacen ni modifican la historia (cit. Turrión Penelas 243), como en Yo mataré monstruos por ti de Santi Balmes en la que aunque se elige el sexo del personaje, la historia no cambia. La personalización se puede realizar de distintas maneras: eligiendo el personaje, como ocurre en The land of mestory time; personalizando la historia con la foto del lector; eligiendo el momento en que se quiere que se desarrolle, el día o la noche, como en Olivia Dreams e incluso la voz y el texto, como en la colección del Ratón Miko, una mezcla entre aplicación de lectura y storytelling.

La personalización puede afectar también al idioma en el que se lea o escuche el libro, como en Mis cuentitos, en que puedes elegir el idioma del texto o de la locución o solo uno de ellos, como es el caso de El baúl de los Monstruos.

\section{Reconocimiento de objetos (vocabulario)}

En este caso, al tocar un objeto aparece su nombre y su pronunciación, un enriquecimiento especialmente útil en el aprendizaje de la lectoescritura. Es lo que ocurre en la aplicación Hugless Douglas, mención especial en la modalidad de ficción de la Feria de Bolonia en 2016.

\section{Efectos de animación del texto}

Al igual que objetos y personajes, el texto puede enriquecerse también con diferentes efectos. Los más habituales son:

Karaoke: Consiste en destacar el texto a medida que se va desarrollando la locución. Un ejemplo de esta opción es The tale of Peter Rabbit de Beatriz Potter, o The Little Which, en la que cada vez que habla un personaje el color de la letra cambia dentro del efecto karaoke.

Movimiento del texto: este movimiento puede darse de diferentes modos, apareciendo en la pantalla como si se tratara de los títulos de crédito de una película, como ocurre en los libros de la aplicación Kidint; apareciendo a medida que se desarrolla la locución, como en Buen provecho. Animales al acecho; desapareciendo el texto una vez leído, como en Lincoln o dejando solo la imagen, como en Blanco Perfecto. 


\section{Otros efectos}

La tecnología se va adaptando a la historia y en algunos casos se consiguen efectos novedosos, por ejemplo: el "efecto vaho", que se limpia al pasar el dedo por la pantalla en Cadavercita Roja, pintar y cambiar el color de las imágenes al pasar el dedo como en el Príncipe Feliz de la aplicación iWilde o el efecto borrador de la mencionada El Hada de los sueños y de Teddy day.

Es necesario mencionar igualmente los efectos de pase de página como un libro convencional, como el telón de un teatro, con la pantalla en negro que desaparece, con el logo de la aplicación...

\section{Lectura y juego}

Aunque no se pueda considerar exactamente como un enriquecimiento, la gamificación es, junto con la personalización, una de las tendencias de peso en los contenidos digitales para niños. La gamificación se define como el uso de estrategias, modelos, dinámicas, mecánicas y elementos propios de los juegos en contextos ajenos a éstos, con el propósito de transmitir un mensaje o unos contenidos o de cambiar un comportamiento, a través de una experiencia lúdica que propicie la motivación, la implicación y la diversión (Gallego, Molina y Llorens, 2).

La gamificación es cada vez más habitual en las aplicaciones de lectura y libros app para niños, y para ello se pueden utilizar varios recursos:

- Incorporación de preguntas relacionadas con la lectura de un título, cuando la resolución de estas preguntas permite obtener premios, puntos, insignias, etc. y la creación de un ranking de lectores.

- Inclusión de juegos y actividades en la propia aplicación, puzles, juegos de memoria, pintura, etc.

- Crear juegos a partir de un libro ya editado. Es el caso de Rita la lagartija, basado en el libro app del mismo título, My Caterpillar ${ }^{8}$, un juego que tiene como protagonista el conocido personaje de Eric Carle, Twit or Miss", basado en el libro "Los Cretinos" de Roald Dahl, Grúfalo Games que tienen como protagonista al personaje creado por Julia Donalson, Emma by Jutta Bauer del libro Jutta Bauer Emma come, Peter Rabbit desarrollado a partir del libro de Beatriz Potter. 


\section{CONCLUSIONES}

La llegada de la lectura digital ha traído consigo algunas dudas, no solo relativas a su calidad, sino también a la capacidad de concentración, a los daños que las pantallas retro-iluminadas puedan provocar en la visión así como a sus ventajas e inconvenientes. Sin embargo, pese a que son temas que por supuesto se deben debatir, consideramos que nuestra preocupación no debe ser tanto el formato, como saber distinguir que el problema, tal como afirma Gemma Lluch ${ }^{9}$ en su blog, no está en la lectura en un formato o en otro sino en la lectura en general y qué hacemos cada uno de nosotros para que sea rica, variada y accesible a cualquier tipo de lector La llegada de las tabletas, ha hecho que el mercado de las aplicaciones aumente constantemente y que dentro de este campo, las apps para niños tengan un peso específico en lo que a popularidad y volumen de ventas se refiere. Las apps se han convertido en un campo especialmente fértil para la LIJD y aunque el negocio de los contenidos digitales infantiles está más desarrollado en los países anglosajones, en España son cada vez más las empresas que están poniendo en marcha proyectos innovadores que están obteniendo reconocimiento internacional, al tiempo que muchos de los productos publicados inicialmente en inglés, se están traduciendo a nuestro idioma.

Este desarrollo de la literatura digital infantil hace más necesaria que nunca la aparición de estudios que ayuden a conocer y entender la nueva realidad. Padres, profesores y bibliotecarios necesitan descripciones y clasificaciones que les permitirán posteriormente seleccionar los contenidos más adecuados y así reducir la brecha entre la atracción de los niños por estos nuevos productos y el desconocimiento de los mismos por parte de todos aquellos implicados en el fomento de la lectura, padres, profesores y bibliotecarios.

Clasificar la LIJD es algo complicado debido no solo a la gran variedad de contenidos, sino también a las múltiples relaciones entre ellos y la continua variabilidad del mercado que hace que continuamente se incorporen nuevos desarrollos, por lo que toda propuesta de clasificación requiere una actualización prácticamente constante.

9 http://www.gemmalluch.com/esp/\%EF\%BB\%BF\%EF\%BB\%BFpero-les-gustala-lectura-en-papel-o-en-pantalla/ 


\section{BIBLIOGRAFÍA}

Alonso-Arévalo, J. y J. A. Cordón-García. "El libro como sistema: hacia un nuevo concepto de libro". Cuadernos de documentación multimedia vol. 26 (2015) http://revistas.ucm.es/ index.php/CDMU/article/download/50628/47030Cahill, Maria, y Anne McGill-Franzen. "Selecting "App" Ealing and "App" Ropriate Book Apps for Beginning Readers." The Reading Teacher 67.1 (2013): 30-39.

Cahill, M., \& A. McGill-Franzen. (2013). “Selecting 'App' ealing and 'App' ropriate Book Apps for Beginning Readers”. Reading Teacher 67(1): 30-39

Cassany, Daniel. En_linea. leer y escribir en la red. Anagrama, 2012.

Escandell Montiel, Daniel. "El libro en la pantalla: hacia un nuevo ensayo en el siglo XXI con la escritura y edición digital". Humanidades Digitales: una aproximación transdisciplinar Janus, Anexo 2 (2014): 73-83.

Gallego Durán, Francisco, Rafael Molina Carmona y Faraón Llorens Largo. “Gamificar una propuesta docente. Diseñando experiencias positivas de aprendizaje”. XX Jornadas sobre la enseñanza universitaria de la informática. https://rua.ua.es/dspace/bitstream/10045/39195/1/ Gamificacio $\%$ CC $\% 81$ n\%20(definicio\%CC\%81n).pdf

García-Rodríguez, Araceli y Raquel Gómez-Díaz. "Las demasiadas aplicaciones: parámetros e indicadores las Topapp de lectura para niños". Anales de Documentación 18.2 (2015). http://dx.doi.org/10.6018/analesdoc.18.2.227071.

"Niños y apps: aprendiendo a leer y escribir en digital". Álabe [En línea], 0.13 (2016): http://revistaalabe.com/index/alabe/article/view/327/232

"Niños y lectura digital: Dispositivos, aplicaciones y contenidos". Barcelona: UOC; El profesional de la información, 2016.

Gómez-Díaz, Raquel, et al. Leyendo entre pantallas. Gijón: Trea, 2016.

Joan Ganz Cooney Center Apps en familia: Guia para usar apps con tus hijos. (2015). http:// www.joanganzcooneycenter.org/wp-content/uploads/2015/08/jgcc_appsenfamilia.pdf K

Koskimaa, Raine. “¿Qué es la literatura digital? Una panorámica general de la literatura digital: de los archivos de texto a los e-books". Textualidades electrónicas-Nuevos escenarios para la literatura. Barcelona: Editorial UOC (Universitat Oberta de Catalunya) (2005): 81-94.

Lebard, Josephine y Guillaume Long, (2015) Le petit guide de la lecture numérique. Montrouge; Bayard Presse $<$ http://liseuses.jaimelirestore.com/INTERNE/Guide_parents/ Guide_Numerique_WEB/WebReader.html>

Lluch, Gemma. "Pero, ¿les gusta la lectura en papel o en pantalla?”. Blog Gemma Lluch. 15 de octubre de 2015. http://www.gemmalluch.com/esp/\%EF\%BB $\%$ BF\%EF\%BB $\%$ BFperoles-gusta-la-lectura-en-papel-o-en-pantalla/

Lucía Mejías, José Manuel. Elogio del texto digital: claves para interpretar el cambio de paradigma. Fórcola Ediciones, 2012.

Manresa Potrony, Mireia. "Leer en digital: la interpretación del lector". Actas del Simposio Internacional Literatura en pantalla: textos, lectores y prácticas docentes. Grupo GRETEL: 26-36. 2014. ISBN: 978-84-942706-4-2. 
Meyers, Eric M, Ehlam Zaminpaima y Aline Frederico. "The Future of Children's Texts: Evaluating Book Apps as Multimodal Reading Experiences". iConference 2014 Proceedings (2014). doi:10.9776/14312

Morales, Isabel. Ciberliteratura. Diccionario de nuevas formas de lectura y escritura. Badajoz: Red Internacional de Universidades Lectoras, 2013. http://dinle.usal.es/searchword. php?valor=ciberliteratura

Ruiz-Domínguez, María del Mar. "Nuevas formas de la literatura infantil: del libro impreso a las aplicaciones digitales". Impossibilia 8 (2014). http://ojs.impossibilia.org/index.php/ impossibilia/article/view/109/83

Sainz, R. M. (Ed.). Realidad Aumentada: Una nueva lente para ver el mundo. Madrid: Fundación Telefónica y Editorial Ariel, 2011.

Turrión Penelas, Celia. "Narrativa infantil y juvenil digital. ¿Qué ofrecen las nuevas formas al lector literario". Tese (Doutorado em Didática da Língua e da Literatura)-Faculdade de Ciências da Educação, Universidade Autônoma de Barcelona, Barcelona, 2014. Print.

Yokota, Junko, y William H. Teale. "Picture books and the digital world." The Reading Teacher 67.8 (2014): 577-585. DOI: 10.1002/trtr.1262 https://www.researchgate.net/ publication/261925043_Picture_Books_and_the_Digital_World

\section{APLICACIONES}

- ¿Quién habrá robado la luna? Interactive Edition. [Libro-app] versión 1.6. 16/05/2015. Disponible en itunes.apple.com.

- A la cama. Dada Company. [Libro-app] versión 2.6. 21/01/2015. Disponible en itunes.apple.com. También disponible en Google Play.

- $\quad$ Alice for the iPad. Atomic Antelope [Libro-app]. versión 4. 26/03/2015. Disponible en itunes.apple.com.

- $\quad$ Animation Studio. miSoft, LLC. [App Storytelling] versión 3.6.0. 4/02/2016 febrero de 2016. Disponible en itunes.apple.com

- Audiobook-Kids. Audiobook Pop. [App audiolibros]. Versión 4.1. 14/12/2012. Disponible en itunes.apple.com. También disponible en Google Play.

- Aurasma. Aurama. [Storytelling/RA]. Versión 5.0.4 07/06/2016. Disponible en itunes.apple.com. También disponible en Google Play.

- Blanco Perfecto. Programaria [Libro-app]. versión 1.0.3. 7/03/2015. Disponible en itunes.apple.com.

- BlueFire. Bluefire Productions [App de lectura]. Versión 2.5. 11/01/2016. Disponible en itunes.apple.com. También disponible en Google Play

- Booksy. Tipitap [App de lectura]. Versión 3.0. 29/09/2014. Disponible en itunes. apple.com. Versión 2.2. 19/04/2014. También disponible en Google Play.

- Buen provecho. Animales al acecho. CIDCLI. [Libro-app]. versión 1.2. 6/12/2013. Disponible en itunes.apple.com. También disponible en Google Play. 
- Buenas noches Mo. Storytoys [Libro-app]. versión 1.0.5. 14/10/2014. Disponible en itunes.apple.com. Versión 1.0.4. 14/10/2014. Disponible en Google Play

- Buenas noches. Fox \& Sheep. [libro-app]. versión 4.2. 9/004/2016. Disponible en itunes.apple.com. Versión 1.3.17. 06/09/2015. También disponible en Google Play.

- Cadavercita Roja. Itbook Editorial Slne. [Libro-app]. versión 1.5. 10/11/2015. Disponible en itunes.apple.com.

- Chaguipatrulla. Storytoys. [Libro-app]. Versión 1.0.9. 24/07/2015. Disponible en itunes.apple.com.

- Comic Marker. Edss Global Company, 2013. [Comic maker]. Versión 2.3.1. 08/05/2015 Disponible en itunes.apple.com.

- Comic Star. Oliver Rice, 2014. [Comic maker]. Versión 1.0.2. 19/10/2014 Disponible en itunes.apple.com.

- Conoce a los animales. Baibuk. Aplicación para acceder al libro homónimo con RA. Disponible en itunes.apple.com y en Google Play.

- Cuentacuentos para niños. Savivo. [Comic maker]. Versión 1.6. 18/11/2014. Disponible en itunes.apple.com También disponible en Google Play.

- Dahl audio. Penguin. [App audiolibros]. Versión 3.0. 22/02/2016. Disponible en itunes.apple.com.

- Descubriendo las telecomunicaciones con Mobi y Fono. Telefónica Soluciones. [Libro app]. Versión 1.1.0. 03/08/2015. Disponible en itunes.apple.com. V También disponible en Google Play.

- $\quad$ Easy Stop Motion. Edoki Academy. [App Storytelling]. Versión. 3.2.9. 15/04/2016. Disponible en itunes.apple.com.

- $\quad$ El árbol de Navidad. Baibuk. [Libro app RA]. Versión 1.1.20/10/2015. Disponible en itunes.apple.com. También disponible en Google Play.

- El Baúl de los monstruos. ItBook [App lectura]. Versión 1.3. 02/03/2013 Disponible en itunes.apple.com.

- El cabeza de Chancho: leyenda de Valparaíso. Cangrejo Ideas. [Libro app]. Versión 1.0. 22/10/2014. Disponible en itunes.apple.com. 16/10/2014. También disponible en Google Play.

- $\quad$ El Hada de los sueños. Mr. Sandan. Hocusbookus. [Libro app]. Versión 1.3. 08/10/2013. Disponible en itunes.apple.com.

- El reino subterráneo. Visual Baker. [Libro app]. Versión 1.2. 01/04/2013. Disponible en itunes.apple.com.

- Emma by Jutta Bauer. Carlsen Verlag Gmb. [App juego]. Versión. 2.4.1. 19/06/2015. Disponible en itunes.apple.com. Basado en el libro Emma come de Jutta Bauer.

- Genius Books. Thaily Cristina Rodrigues Cadeffo. [App de lectura]. Versión 6.1. 08/10/2013. Disponible en itunes.apple.com.

- Gruffalo Games. Magic Light Pictures. [App juego]. Versión 1.0.1. 18/09/2014. Disponible en itunes.apple.com. Basada en el libro del mismo título de Julia Donalson. 
- Hansel y Gretel. BooksARalive [Libro app RA]. Versión 1.0. 13/10/2015. Disponible en itunes.apple.com. También disponible en Google Play.

- Hugless Douglas. Bookpoint. [Libro app]. Versión 2.0.2. 11/01/2012. Disponible en itunes.apple.com.

- Ibooks. [App de lectura] Disponible en itunes.apple.com.

- $\quad$ iDinosaurio. Red Frog Digital Limited. [Libro app RA]. Versión 2.3.1. 11/02/2016. Disponible en itunes.apple.com. También disponible en Google Play.

- Imagistory. Imagistory Publishing. [App storytelling]. Versión 1.2.1. 29/10/2014. Disponible en itunes.apple.com.

- Imagnotron. Moonbot Studios. [RA] Versión 1.0.3. 28/07/2012

- In my dream. e-Toiles Editions. [Libro app]. Versión 1.3.1. 29/01/2014. Disponible en itunes.apple.com. Disponible en bundle e-Toiles box.

- Jack-o-Lantern. Baibuk [Libro app AR]. Versión 1.0. 06/11/2013. Disponible en itunes.apple.com. También disponible en Google Play.

- Joy Tales. Desdoo. [App de lectura]. Versión 1.4.0. 04/12/2013. Disponible en itunes.apple.com.

- $\quad$ Kidint. Soluciones Tecnológicas e Informáticas. [App de lectura]. Versión 2.1.1. 03/06/2016. Disponible en itunes.apple.com. También disponible en Google Play.

- Kindle. AMZN Mobile LLC. [App de lectura]. Versión 4.20. 16/05/2016. Disponible en itunes.apple.com. También disponible en Google Play.

- Las hadas de la casa Lis. Chocosoft. [Libro app]. Versión 1.1. 01/06/2012. Disponible en itunes.apple.com. También disponible en Google Play.

- Lincoln. Quelle Histoire. [Libro app]. Version 1.0.3. 06/10/2015. Disponible en itunes.apple.com.

- Lo que comen los ratones. eMutation New Media. [Libro app]. Versión 1.0.3. 06/10/2015. Disponible en itunes.apple.com

- Los fantásticos libros voladores de Mr. Morris Lessmore. Moonbot Studios. [Libro app]. Versión 1.4.5. 13/08/2015. Disponible en itunes.apple.com.

- Love to read: tales of Beatrix Potter. Onebillion Children. [App de lectura]. Versión 1.0. 27/05/2014. Disponible en itunes.apple.com.

- Make Beliefs Comix. Guarionex Press. [Cómic marker]. Versión 1.4. 06/10/2015. Disponible en itunes.apple.com. También disponible en Google Play.

- Medusa! HD. Jamie McBride. [Libro app]. Versión 2.0. 21/05/2014. Disponible en itunes.apple.com.

- $\quad$ Mi vecino de abajo. Mobile Republic. [Libro app]. Versión 1.0. 20/03/2011. Disponible en itunes.apple.com. Basado en el libro del mismo título de Daniel Nesquens

- Miko (colección). Auryn. [Libro app/Storytelling]. Versión 1.0.2. 04/02/2013. Disponible en itunes.apple.com. También disponible en Google Play.

- Mis cuentitos. Pam Digital. [App de lectura]. Versión 1.0.5. 01/12/2014. Disponible en itunes.apple.com. 
- $\quad$ My Caterpillar. StoryToys Entertainment. [App juego]. Versión 1.0.6. 16/03/2014. Disponible en itunes.apple.com. Basado en el libro Mi pequeña oruga glotona de Eric Carle. También disponible en Google Play.

- $\quad$ Nurot. Programaria. Libro app]. Versión 1.0.4. 24/03/2016. Disponible en itunes. apple.com

- $\quad$ Olivia Dreams. DreamWorks Animation. [Libro app]. Versión 1.1.0. 07/11/2014. Disponible en itunes.apple.com.

- $\quad$ Peter Rabbit. Endles Text Runner of the Beatrix Potter Clasic Tale. Kevin Ayre. [App juego]. Versión 1.2. 30/04/2016. Disponible en itunes.apple.com. Basado en el libro del mismo título de Beatrix Potter.

- $\quad$ Play Kids. Movile Internet Movel. [App videolibros]. Versión 3.3.4. 03/06/2016. Disponible en itunes.apple.com. También disponible en Google Play.

- $\quad$ Play Tales. Play Tales. [App de lectura]. Versión 2.12. 24/09/2013. Versión suscripción Play Tales Gold. 1.9.3. 09/11/2014. Disponible en itunes.apple.com.

- Por cuatro esquinitas de nada. Dada Company [Libro app]. Versión 2.8 18/12/2014 También disponible en Google Play.

- $\quad$ Rita la lagartija. Irene Blasco Grau. [Libro app]. Versión 1.1. 09/12/2012. [App juego].Versión 1.1. 19/12/2012. Disponible en itunes.apple.com

- Roxie's Doors. OC Graphics. [RA] Versión 1.6. 16/01/2013. Disponible en itunes. apple.com. También disponible en Google Play.

- Sesame Street eBook. Sesame Workshop Apps. [App de lectura]. Versión 1.6. 18/10/2012. Disponible en itunes.apple.com. También disponible en Google Play.

- $\quad$ StoriesAlive. Auryn. [App de lectura y app videolibros]. Versión 4.1.7. 05/02/2015. Disponible en itunes.apple.com. También disponible en Google Play.

- Story Time. Teknowledge Software. [App de lectura]. Versión 3.0. 05/11/2014. Disponible en itunes.apple.com.

- Strip Designer. Vivid Apps. [Comic marker]. Versión 1.18.3. 05/06/2016. Disponible en itunes.apple.com.

- Teddy’s Day. Auryn. [Libro app]. Versión 3.0.0. 31/05/2014. Disponible en itunes. apple.com.

- $\quad$ The land of mestory time. Made in mi. [Libro app] Versión 0.0.4. 08/12/2012. Disponible en itunes.apple.com.

- The Little Which. Slimcriket. [Libro app]. Versión 4.0.0. 31/05/2016. Disponible en itunes.apple.com

- $\quad$ The tale of Peter Rabbit. Penguin Books. [Libro app]. Versión 1.2. 31/03/2016. Disponible en itunes.apple.com.

- $\quad$ Toontastic Jr. Pirates. Launchpad Toys. [App storytelling]. Versión 1.5.2. 04/02/2015. Disponible en itunes.apple.com

- Toontastic. Launchpad Toys. [App storytelling]. Versión 2.15.2. 04/02/2015. Disponible en itunes.apple.com. 
- $\quad$ Twit or Miss. Penguin Books. [App juego]. Versión 1.2.1. 09/1172015. Disponible en itunes.apple.com. También disponible en Google Play. Basado en el libro "Los cretinos" de Roald Dahl.

- Un lobo boquiabierto. Paidotribo. [Libro app RA]. Versión 2.0. 02/06/2016. Disponible en itunes.apple.com.

- Vivaz Books. Evaristo Vinuesa Rivera; Vivaz Books. [App videolibros]. Versión 1.3.4. 27/11/2013. Disponible en itunes.apple.com.

- World of Dinnosaurs: Kids Appersian. [Libro app]. Versión 2.1. 12/05/2016. Disponible en itunes. También disponible en Google Play.

- Wuwu \& Co. Step in Books ApS. [Libro app]. Versión 1.5. 08/05/2014. Disponible en itunes.apple.com. Versión 2. 17/02/2016. También disponible en Google Play.

- Yo mataré monstruos por ti. Raúl Soler. [Libro app]. Versión1.5.08/05/2014. También disponible en Google Play. 